\title{
PREVENCIÓN E INTERVENCIONES TEMPRANAS EN SALUD MENTAL: UNA PERSPECTIVA INTERNACIONAL ${ }^{1}$
}

\begin{abstract}
Matías Irarrázaval ${ }^{2}$, Fernanda Prieto $^{3}$, Julio Armijo ${ }^{4}$
Resumen: Las enfermedades mentales son comunes, crónicas y discapacitantes, y representan un problema de salud pública importante en nuestro país y en todo el mundo. Para las enfermedades mentales más comunes, disponemos actualmente de intervenciones médicas y psicosociales con eficacia probada. Sin embargo, estas aún son insuficientes, y su disponibilidad no se condice con la alta morbilidad y mortalidad que encontramos para las enfermedades mentales. En respuesta a esto, en los últimos 15 años se ha realizado un esfuerzo considerable para desarrollar técnicas de detección temprana, para personas con alto riesgo clínico de desarrollo de enfermedad mental, e intervenciones precoces para prevenir o retrasar la progresión a la enfermedad completa. Un mayor énfasis en la salud mental y la integración de la atención a la salud mental en todos los programas de desarrollo pertinentes, fortalecerán el esfuerzo general de desarrollo, así como garantizará, por primera vez, la atención sostenida de la inversión y la investigación al principal contribuyente mundial de la pérdida de productividad y carga de enfermedad.
\end{abstract}

Palabras clave: prevención, intervención temprana, salud mental

Prevention and early interventions in mental health: an international perspective

\begin{abstract}
Mental diseases are common, chronic and disabling, representing a problem in public health of importance in our country and in the world. For most common mental diseases, currently we dispose of medical and psycho social interventions with proven efficacy. Nevertheless, these are not sufficient yet, and their availability does not diminish the morbidity and mortality produced by mental diseases. In order to solve this, a considerable effort has been carried out in the last 15 years to develop techniques of early detection for persons with high clinical risk to develop mental illness and precocious interventions to prevent or delay the progression of full illness. A greater emphasis in mental health and integrating mental health care in all relevant development programs will strengthen the general development effort, as well as it will ensure, for the first time, sustained attention to investment and research to the main world contributor to loss of productivity and disease burden.
\end{abstract}

Key words: prevention, early intervention, mental health

Prevenção e intervenções precoces em saúde mental: uma perspectiva internacional

Resumo: As enfermidades mentais são comuns, crônicas e descapacitantes, e representam um problema de saúde pública importante em nosso país e em todo o mundo. Para as enfermidades mentais mais comuns dispomos atualmente de intervençôes médicas e psicossociais com eficácia comprovada. Entretanto, estas ainda são insuficientes, e sua disponibilidade não condiz com a alta morbidade e mortalidade que encontramos para as enfermidades mentais. Em resposta a isto, nos últimos 15 anos foi realizado um esforço considerável para desenvolver técnicas de detecçáo precoce para pessoas com alto risco clínico de desenvolvimento de enfermidade mental, e intervençóes precoces para prevenir ou atrasar a progressáo da enfermidade completa. Uma maior ênfase na saúde mental e a integração da atenção à saúde mental em todos os programas de desenvolvimento pertinentes, fortalecerão o esforço geral de desenvolvimento, assim como garantirá, pela primeira vez, a atenção sustentada no investimento e na pesquisa do principal contribuinte mundial da perda de produtividade e carga de enfermidade.

Palavras-chave: prevenção, intervenção precoce, saúde mental

\footnotetext{
${ }^{1}$ Financiado por el proyecto FONDECYT No 11130615, mediante la Comisión Nacional de Investigación Científica y Tecnológica (CONICYT).

${ }^{2}$ Departamento de Psiquiatría, Hospital Clínico, Facultad de Medicina, Universidad de Chile, Santiago, Chile. Instituto Milenio para la Investigación en Depresión y Personalidad, Santiago, Chile

Correspondencia: mirarrazavald@u.uchile.cl

${ }_{3}^{3}$ Departamento de Psiquiatría, Hospital Clínico, Facultad de Medicina, Universidad de Chile, Santiago, Chile

${ }^{4}$ Equipo de Psiquiatría Social y Comunitaria, Clínica Psiquiátrica Universitaria, Santiago, Chile
} 


\section{Introducción}

Hace 100 años, la atención en salud estaba centra$\mathrm{da}$ en las enfermedades infecciosas agudas(1). Las enfermedades crónicas infecciosas y no infecciosas, como la tuberculosis, la sífilis, la lepra y las enfermedades mentales, con frecuencia estaban limitadas a los grandes hospitales o instituciones públicas, donde los tratamientos eran, en muchos casos, la privación de la libertad o la institucionalización. Actualmente, en Chile y en la mayor parte de los países desarrollados, las enfermedades infecciosas agudas son, en su mayoría, prevenibles y/o curables. Nos encontramos en la era de la enfermedad crónica.

Muchas de las enfermedades crónicas de principios del siglo XX, como la lepra, la sífilis y hasta hace poco la tuberculosis y la poliomielitis, han llegado a ser tan raras como desconocidas para las nuevas generaciones de médicos. La excepción notable en esta historia de progreso es la enfermedad mental. A pesar de los nuevos medicamentos y tratamientos psicosociales, las enfermedades mentales, como la esquizofrenia y los trastornos del ánimo, son un problema importante de salud pública, prácticamente de la misma forma que lo eran hace un siglo. Las enfermedades mentales son reconocidas cada vez más como enfermedades crónicas y discapacitantes, que se asemejan, desde el punto de vista de su gravedad y costo, a las enfermedades cardiovasculares, el cáncer y la diabetes. Aun así, los investigadores continúan considerando el tratamiento de las enfermedades mentales como diferente de otras enfermedades médicas. Mientras los objetivos de la investigación biomédica para la enfermedad cardiovascular, la diabetes y el cáncer en general son la cura y la prevención, comparativamente existe poca investigación acerca de estos aspectos para la enfermedad mental. A pesar de lo anterior, la investigación en enfermedad mental ha tenido avances exponenciales durante las últimas décadas.

Considerando lo anterior, la pregunta que debemos hacernos es: ¡cuál es una estrategia óptima para disminuir la prevalencia de la enfermedad mental? ¿Cómo podemos asegurar que en menos de 100 años la esquizofrenia, los trastornos del estado de ánimo y el autismo se unan a la lepra, la sífilis y la tuberculosis como enfermedades de baja frecuencia?

\section{El reto para la salud global}

Como punto de partida, es importante reconocer el desafío. Las enfermedades mentales son comunes, crónicas y discapacitantes. Una revisión metaanalítica de 174 encuestas sobre salud mental en 63 países diferentes mostró que aproximadamente 1 de cada 5 personas $(17,6 \%$, IC 95\%: 16,3$18,9)$ habían tenido una enfermedad mental común en los últimos 12 meses, y un 29,2\% (IC 95\%: 25,9-32,6) de los encuestados refirieron haber presentado una enfermedad mental en algún momento de sus vidas. Se encontró un efecto de género consistente: las mujeres presentaron tasas más altas de trastornos del ánimo $(7,3 \%: 4,0 \%)$ y ansiedad (8,7\%:4,3\%) que los hombres durante los últimos 12 meses. Ellos, en cambio, presentaron tasas mayores para trastornos por uso de sustancias $(2,0 \%: 7,5 \%)$, con un patrón similar para las prevalencias de vida. También se encontró una variación regional consistente. Los países del norte y sureste de Asia mostraron prevalencias del último año y de vida sistemáticamente inferiores que otras regiones. Las tasas de prevalencia del último año también fueron bajas en el África subsahariana, mientras que en los países anglosajones las prevalencias estimadas son las más altas en términos globales(2).

En Chile, casi una quinta parte de la población adulta $(19,7 \%)$ y más de un cuarto de la población infanto-adolescente $(22,5 \%)$ ha tenido un trastorno psiquiátrico durante el último año $(3,4)$. Los trastornos más frecuentes en los adultos, en los últimos 6 meses, son la fobia simple, fobia social, agorafobia, trastorno depresivo mayor y la dependencia del alcohol. En cambio, en niños y adolescentes, los trastornos más frecuentes son el trastorno por déficit de atención, los trastornos conductuales y los trastornos ansiosos. No solo es relevante la alta prevalencia de la enfermedad mental, sino también las dificultades que tienen los pacientes para acceder y conseguir tratamiento. Menos del $40 \%$ de las personas con algún diagnóstico psiquiátrico en Chile han recibido un tratamiento durante los últimos seis meses. En Latinoamérica las cifras son similares (39\%), siendo menor para algunas patologías como el abuso o dependencia de alcohol (24\%)(5).

En un intento de cuantificar la carga de cada una 
de las principales enfermedades para la salud pública y la sociedad, durante los ańos noventa la Organización Mundial de la Salud lanzó el estudio sobre la Carga de Enfermedad. Este estudio, que utiliza los "años de vida ajustados por discapacidad” para considerar la duración así como la gravedad de la discapacidad, identificó al grupo de las enfermedades mentales como la primera causa de años de vida perdidos por discapacidad entre todas las enfermedades médicas(6). A diferencia de otras causas importantes de morbilidad y mortalidad, como las enfermedades cardiovasculares, el cáncer y los accidentes cerebrovasculares, las enfermedades mentales son crónicas y comienzan a temprana edad. Un 70\% de las enfermedades mentales comienzan antes de los 18 años de edad y suelen mantenerse por décadas $(7,8)$.

Es evidente que estas enfermedades representan una mortalidad considerable. Existen aproximadamente 800.000 suicidios cada año en el mundo (1.500 en Chile, siendo la mayor tasa de Latinoamérica), con un $90 \%$ de ellos relacionados con una enfermedad mental. En el ámbito global esto es mayor que el número de muertes por homicidio (500.000) y debido a guerras (350.000)(9).

Mientras que el suicidio se asocia generalmente con la depresión, el riesgo relativo de muerte por suicidio se incrementa casi 10 veces en la esquizofrenia y 50 veces en la anorexia nerviosa(10).

Tal vez lo más sorprendente en el estudio de los datos de morbilidad y mortalidad es darnos cuenta que hemos avanzado tanto en reducir la prevalencia de la enfermedad cardiovascular, el SIDA, ciertos tipos de cáncer, e incluso el homicidio, y sin embargo tan poco en disminuir las tasas de los trastornos mentales más frecuentes y el suicidio. Globalmente las tasas de suicidio han aumentado persistentemente desde los años $50 \mathrm{y}$, aunque no se ha observado un aumento en las tasas de enfermedad mental, los números totales han aumentado en respuesta a los cambios en la estructura poblacional $(11,12)$.

Con relación a la morbilidad, los datos epidemiológicos no son alentadores. Es evidente que durante la última década más personas están recibiendo tratamiento para las enfermedades mentales, pero hay poca evidencia hasta la fecha que esto se acompañe de una reducción de la discapacidad(13). De acuerdo con la Organización Mundial de la Salud, más mil millones de personas - que corresponden a un $15 \%$ de la población total del planeta- se encuentran en situación de discapacidad. Cifra que aumenta año a año, debido al envejecimiento de la población y al aumento de las enfermedades crónicas (66,5\% de todos los ańos vividos con discapacidad en los países de ingresos bajos y medianos)(14). Se afirma que las personas con discapacidad tienen menos acceso a los servicios de asistencia sanitaria $y$, por lo tanto, necesidades insatisfechas en relación con ello(15). En relación a la salud mental, se calcula que existen 450 millones de personas en el mundo que sufren actualmente de trastornos mentales, y que el gasto generado por estos puede equivaler al 3-4\% del producto nacional bruto de los países desarrollados(16). Si entendemos por discapacidad la limitación producida por una deficiencia física o mental al interactuar con las barreras del entorno(17), nuestro desafío es levantar las barreras que tienen los pacientes para acceder y conseguir tratamiento, así como todas aquellas barreras (educacionales, laborales, de vivienda y alojamiento, entre otras) que generen discapacidad mental.

\section{¿Estamos progresando?}

La limitada evaluación sobre la salud mental global que se ha realizado anteriormente no considera un progreso importante en diversas áreas. Para las enfermedades mentales más comunes disponemos actualmente de intervenciones médicas y psicosociales con eficacia probada en ensayos controlados aleatorios. Entre las excepciones se incluyen la anorexia nerviosa y el autismo, en las que todavía son escasos los ensayos controlados de gran escala que demuestren la eficacia de las intervenciones utilizadas actualmente. A pesar de ello, incluso en enfermedades crónicas y discapacitantes como la esquizofrenia, disponemos de medicamentos que reducen la mayoría de los síntomas positivos, permitiendo en muchos casos que los pacientes puedan abandonar los hospitales e incorporarse a la sociedad. Muchos de los pacientes con trastornos del estado de ánimo responden al tratamiento farmacológico y algunos se recuperan por completo(18). 
Sin embargo, la disponibilidad de tratamientos efectivos no se condice con la alta morbilidad y mortalidad que encontramos para las enfermedades mentales. Entonces, ¿¿cuál es el problema con relación al tratamiento? La respuesta tradicional es que fallamos en la prestación de los servicios, es decir, son pocos los pacientes que reciben las intervenciones basadas en la evidencia. Tenemos evidencia para apoyar este argumento. Un reciente metaanálisis de 28 estudios demuestra que la psicoeducación familiar puede reducir las recaídas en la esquizofrenia en un $50 \%$, pero solo el $31 \%$ de los pacientes recibe esta intervención $(19,20)$.

El estudio PORT demostró que existe una baja tasa de utilización de varios tratamientos basados en la evidencia para esquizofrenia(21). El estudio NCS-R reportó que solo el $50 \%$ de las personas con depresión reciben algún tipo de tratamiento y aproximadamente un $20 \%$ recibe un tratamiento que es mínimamente adecuado(13).

La terapia cognitivo-conductual ha demostrado ser una psicoterapia eficaz para los trastornos del ánimo y de la ansiedad, pero sabemos que la mayoría de los programas de formación de los profesionales en salud mental no proporcionan una formación adecuada en este o cualquier otro tratamiento basado en la evidencia(22). Los estudios han demostrado que incluso las mejores prácticas de tratamiento aplicadas al $100 \%$ de la población con enfermedad mental evitarían solo el $40 \%$ de la carga de la enfermedad(23).

\section{¿Estamos detenidos?}

Si bien sabemos que no hemos utilizado en forma completa los tratamientos disponibles, es importante reconocer que los tratamientos actuales en salud mental aún son insuficientes. Recordando la metáfora del río - utilizada tantas veces en salud pública-, al mismo tiempo que estamos haciendo más y mejores esfuerzos para rescatar a los niños del río, debiésemos también buscar cuál es la causa de que los niños se están cayendo al río, o trabajar río arriba. Todos los tratamientos médicos actuales para las enfermedades mentales son paliativos, y ninguno de estos se ha propuesto como cura. Incluso muy pocas personas reciben cuidados paliativos basados en la evidencia.
Continuando con la metáfora del río, todavía no nos hemos puesto de cabeza a buscar la vacuna. En efecto, mientras que la OMS, en su último Plan de Acción en Salud Mental 2013-2020, proclamó la recuperación como uno de los objetivos de la atención en salud mental, pocos ensayos clínicos han apuntado hacia la "recuperación" como resultado $(24,25)$. Para ser justos, puede ser que los investigadores estén evitando el término "recuperación", porque es difícil de definir y cuantificar. Si bien, desde una perspectiva psicosocial, la recuperación consiste en trabajar con los usuarios para conseguir sus metas personales y mejorar su calidad de vida, independientemente de la evolución de sus síntomas(26), se ha sugerido que en las enfermedades mentales, como en otras enfermedades médicas, debemos aspirar más bien a una meta de recuperación definida como una remisión completa y permanente. En otras áreas de la medicina, este enfoque ha recibido el nombre de "tratamiento curativo". Sin embargo, ¿qué tan eficaz es actualmente? En algunos trastornos, como el autismo y la anorexia nerviosa, simplemente carecemos de tratamientos biomédicos para los síntomas principales. Para otras enfermedades, como la esquizofrenia, los tratamientos actuales se dirigen solamente a disminuir los síntomas. Los fármacos antipsicóticos reducen los síntomas psicóticos, pero no tratan los déficits cognitivos o la disfuncionalidad asociada a la esquizofrenia(27).

En las últimas cinco décadas la investigación ha demostrado que los antipsicóticos tienen mayor eficacia que el placebo para reducir las alucinaciones y los delirios en la esquizofrenia. Sin embargo, la eficacia de estas drogas sigue siendo decepcionante. El estudio CATIE demostró que solo uno de cada cuatro pacientes con esquizofrenia continuó el tratamiento antipsicótico luego de 18 meses de tratamiento(28).

La esquizofrenia sigue siendo una enfermedad crónica y discapacitante, tal como lo era hace un siglo. Sabemos que las intervenciones psicosociales, como el empleo con supervisión y la psicoeducación familiar, reducen las recaídas y pueden ayudar a los pacientes a lograr una vida en la comunidad. Sorprendentemente, aún no podemos saber cuántos o cuáles de los pacientes con esquizofrenia se recuperarán con el uso combinado de las intervenciones médicas y psicosociales 
actuales. Y nuestras expectativas siguen siendo limitadas. Por ejemplo, la definición por consenso sobre la remisión en la esquizofrenia, desarrollada por el grupo de trabajo especializado de la Asociación Americana de Psiquiatría 10 años atrás, ni siquiera incluía en un comienzo el requisito de la mejoría en el funcionamiento, que muchos pacientes y familias ven como la esencia de la recuperación(29).

¿Son los tratamientos para los trastornos del ánimo mucho mejores? Los antidepresivos, la tercera clase de medicamentos más comúnmente utilizados a nivel mundial, han demostrado reducir los síntomas del trastorno depresivo mayor, pero estos fármacos son generalmente mejores que el placebo en un $20-30 \%$ y solo después de seis semanas de tratamiento. Además, en la mayoría de los estudios, los pacientes que toman antidepresivos se mantienen con síntomas después de un tiempo considerable(30).

Se ha definido mediante consenso que la remisión es uno de los objetivos principales en el tratamiento de la depresión. ¿Con qué frecuencia el uso de inhibidor selectivo de la recaptación de serotonina (ISRS) se asocia a la remisión en depresión? En un estudio a gran escala sobre la eficacia del citalopram, menos de un tercio de los pacientes cumplieron con los criterios de remisión luego de 12 semanas de tratamiento(31). El placebo no se utilizó como comparación para este estudio, por lo que no está claro cuántos de estos pacientes pudieron recuperarse sin antidepresivos.

Incluso aceptando que alrededor de un tercio de los pacientes tienen remisión para un tratamiento antidepresivo, ¿es el tratamiento suficiente? En una enfermedad caracterizada por un intenso sufrimiento emocional, desesperanza y frecuente ideación suicida, ¿¿debemos estar satisfechos con tratamientos que requieren varias semanas para ser eficaces y que no necesariamente producirán la remisión en la mayoría de los pacientes? Nuestros colegas en otras especialidades médicas, ¿estarían satisfechos con estos resultados para una enfermedad que afecta al $20 \%$ de la población y que se caracteriza por un sufrimiento intenso, una alta morbilidad y un riesgo de mortalidad tres veces mayor que el resto de la población?
Al considerar las deficiencias de los tratamientos actuales, difícilmente se puede ignorar la falta de progreso en el desarrollo de medicamentos con mecanismos novedosos. A pesar de que durante las últimas décadas se han introducido decenas de nuevos antipsicóticos y antidepresivos, con el objetivo de reducir los efectos secundarios o mejorar la eficacia de los tratamientos, la utilidad de estos "nuevos" medicamentos es limitada, al ser simplemente variaciones de los viejos medicamentos. En contraste con otras áreas de la medicina, en el último tiempo se han desarrollado pocos medicamentos con nuevos mecanismos de acción para la esquizofrenia y la depresión. En efecto, después de más de dos décadas desde que se comenzaron a utilizar, aún no conocemos completamente los mecanismos de acción de todos los medicamentos que utilizamos actualmente, y no es del todo claro si los antipsicóticos atípicos más nuevos o los ISRS representan compuestos con diferentes mecanismos de acción o simplemente análogos químicos con menores efectos adversos(32).

\section{Un enfoque diferente}

Si nos centramos en los beneficios y dificultades del tratamiento en salud mental no hay duda de que necesitamos un enfoque diferente. En las últimas cinco décadas el tratamiento de la enfermedad mental ha sido dominado por el estudio de la acción de los fármacos disponibles. En retrospectiva, esto podría haber sido productivo si los fármacos hubieran atacado la fisiopatología de la enfermedad mental. Ciertamente, el estudio de la acción de la insulina ha sido útil para el desarrollo de nuevos enfoques para el tratamiento de la diabetes y la investigación sobre la farmacología de los lípidos ha sido de utilidad para la medicina cardiovascular. Sin embargo, como acabamos de revisar, los medicamentos disponibles en la psiquiatría son insuficientes para el manejo de los trastornos, y el foco exclusivo en este tratamiento no logrará disminuir la prevalencia de la enfermedad mental.

Es necesario cambiar el enfoque. Uno de los sueńos de los clínicos que se percatan a diario de las limitaciones del tratamiento en salud mental, es poder llegar antes que se desarrolle la enfermedad. La verdad es que ese sueño es posible hoy en día. 


\section{Prevención en salud mental}

Los sujetos que se consideran como de alto riesgo clínico tienen una probabilidad elevada de desarrollar un trastorno psiquiátrico. Estos individuos a menudo se identifican mediante el uso de tres criterios diferentes, aunque frecuentemente algunos cumplen con más de uno. En primer lugar, las personas con síntomas subumbrales están en alto riesgo clínico de una enfermedad mental. Es lo que ocurre con las alucinaciones o delirios en la esquizofrenia o los síntomas del ánimo en la bipolaridad. Por otra parte, los sujetos con familiares de primer grado que tienen una enfermedad mental, así como un reciente deterioro en el funcionamiento también están en alto riesgo clínico. Por último, en algunas patologías, como la psicosis, se han encontrado síntomas breves autolimitados como un factor de riesgo confiable para el desarrollo posterior de la enfermedad(27).

En la esquizofrenia, por ejemplo, el primer estudio publicado sobre la utilización de estos criterios para la identificación de individuos de alto riesgo encontró una tasa de transición a psicosis completa de un $40 \%$ dentro de un año(27). En el caso de la bipolaridad, 20\% de los adolescentes que presentan síntomas subsindromáticos (trastorno afectivo bipolar no especificado) desarrolla trastorno bipolar tipo I después de 4 años de seguimiento(33).

Asimismo, un $23 \%$ de los sujetos con riesgo de depresión durante la adolescencia desarrolla la enfermedad en los años de transición hacia la adultez(34).

También ha habido un aumento relativamente reciente en la detección de factores de riesgo asociados a trastornos neurológicos, como la demencia(35), así como también enfermedades físicas, como la enfermedad cardiovascular(36).

Pueden ser utilizados otros indicadores de vulnerabilidad en forma agregada. Por ejemplo, en sujetos con un alto riesgo de esquizofrenia es frecuente encontrar alteraciones en la percepción, en el pensamiento, altos niveles de suspicacia, disminución de volumen cerebral y diversos déficits cognitivos(37). En la depresión ocurre algo similar, siendo el neuroticismo, los niveles y la respuesta del cortisol diurno, y la disminución de la corteza lateral del hemisferio derecho y medial del izquierdo, endofenotipos que aumentan significativamente el riesgo de desarrollar la enfer$\operatorname{medad}(38)$.

En los últimos 15 años se ha dedicado un esfuerzo considerable al desarrollo de técnicas de detección temprana para personas con alto riesgo clínico de desarrollo de enfermedad mental, e intervenciones precoces para prevenir o retrasar la progresión a la enfermedad completa. Ejemplo de ello son el desarrollo de diversas herramientas de detección, en un intento por predecir de mejor manera el riesgo que tienen los individuos para progresar a la enfermedad mental, que incluyen historias narrativas, baterías de pruebas neuropsicológicas y cuestionarios de evaluación de síntomas, entre otros. Junto con esto, una serie de ensayos clínicos ha examinado la eficacia de la terapias específicas y uso de medicamentos en personas que están en alto riesgo clínico para el desarrollo de enfermedades como la psicosis, la depresión o la bipolaridad(39-41).

El relativamente nuevo énfasis en la intervención temprana para la enfermedad mental ha tenido un impacto significativo en el campo de la psiquiatría, lo que ha llevado incluso a la creación en el DSM-5 de una nueva categoría diagnóstica relacionada, que identifica a las personas que han experimentado síntomas por debajo del umbral de psicosis: el Síndrome de Psicosis Atenua$\mathrm{da}(42)$. Por lo tanto, es evidente que las estrategias de prevención e intervención temprana se han convertido en un foco principal en la investigación en Psiquiatría y Psicología, lo que abre un nuevo abanico de consideraciones éticas para los profesionales de la salud que trabajan en esta área.

En el ámbito de la salud pública se ha encontrado evidencia de la existencia de intervenciones que promueven la salud mental y poseen un impacto en la prevención de la aparición de los trastornos mentales. La evidencia específica del impacto de tales esfuerzos en promoción y prevención no es fácil de determinar, por la calidad de la metodología utilizada; sin embargo, estudios experimentales relevantes han mostrado la efectividad de la prevención en la enfermedad mental. Solo considerando ejemplos en depresión y ansiedad, 
encontramos que, en Chile un programa de ejercicio físico basado en la escuela redujo los síntomas de ansiedad(43). En China, un programa de prevención de la depresión que tuvo como objetivo educar a escolares para el desarrollo de pensamientos positivos, resolución de conflictos y habilidades en la toma de decisiones, mostró eficacia en la reducción de los síntomas depresi$\operatorname{vos}(44)$. Un ensayo clínico dirigido a estudiantes de enfermería iraníes reportó que un programa de orientación educativa de frecuencia semanal reduce la ansiedad en el largo plazo(45).

Otro estudio controlado aleatorizado en Nigeria, de programas de prevención en adultos en situación de riesgo para la depresión y la ansiedad, tales como pacientes quirúrgicos, también mostró efectos beneficiosos(46).

\section{¿Cómo producen su efecto las intervenciones preventivas en salud mental?}

Existe evidencia contundente acerca de la relación entre factores de riesgo y de protección y el desarrollo de las enfermedades mentales(47). Tanto los factores de riesgo como los protectores pueden influir individual, familiar y socialmente, y también en un nivel macro, mediante factores económicos y culturales. Generalmente, la enfermedad mental se produce por el efecto acumulativo de la presencia de múltiples factores de riesgo, la falta de factores protectores y la interacción de situaciones de riesgo y protección, lo que predispone a los individuos a cambiar de una condición mentalmente saludable a una de mayor vulnerabilidad, luego a un problema mental y finalmente a una enfermedad mental con todas sus características.

Por ejemplo, experiencias estresantes en la vida, tales como la exposición a la violencia y la deficiente salud física, están bien reconocidos como factores de riesgo para los trastornos mentales. Es evidente que situaciones sociales adversas, como la pobreza, aumentan el riesgo de enfermedad mental, y la mayoría de los estudios poblaciones muestran consistentemente que las personas pobres y marginadas poseen un mayor riesgo de padecer trastornos depresivos y ansiosos(48). Asimismo, el aislamiento social -independiente del nivel de ingresos- se relaciona con sintomatolo- gía depresiva y fragmentación del sueño, así como con un aumento del riesgo de mortalidad en un periodo de seis años (entre otros, por su relación con mayores cifras de presión arterial y mayor riesgo cardiovascular) (49). También sabemos que el bajo acceso a la posibilidad de atención en el sistema público produce, en algunos países, que los individuos busquen proveedores de salud privados con el consiguiente "gasto de bolsillo" y pérdida de las oportunidades de empleo.

Por otro lado, conocemos la relación entre la enfermedad mental y la mortalidad infantil. Una serie de estudios realizados en el sur de Asia han demostrado que los retrasos del desarrollo y la desnutrición en niños menores de un año se asocia con la depresión materna en forma independiente(50).

Un estudio poblacional de cohorte en Pakistán mostró que los bebés de madres que estaban deprimidas durante el embarazo y en el periodo posnatal tenían cinco veces más riesgo de tener bajo peso y retraso en el crecimiento a los seis meses que los bebés de madres no deprimidas, incluso después de ajustar por otros factores confundentes conocidos, tales como el nivel socioeconómico de la madre(51).

El fracaso del desarrollo durante la niñez es un factor de riesgo importante para la mortalidad infantil; por lo tanto, sería plausible la hipótesis que la depresión en las madres también se asocia con un aumento de la mortalidad infantil. En efecto, la evidencia muestra que las madres con depresión tienen más probabilidades de abandonar la lactancia materna, y que sus bebés son significativamente más propensos a sufrir episodios de diarrea o a no tener sus vacunas completas, todos ellos factores de riesgo reconocidos para la mortalidad infantil. Este estudio también mostró que la depresión durante el embarazo se asocia fuertemente con un bajo peso al nacer, una vinculación que ha sido replicada en estudios en la India y Brasil(52). Finalmente, estudios recientes han demostrado cómo la depresión perinatal aumenta en un 1.28 veces el riesgo de depresión mayor en el hijo a la edad de 18 años (IC 95\% 1.08-1.51, p=0.003). Su tratamiento oportuno y efectivo podría incluso contribuir a la disminución de la prevalencia de depresión de nuevas generaciones(53). 
Las intervenciones para prevenir los problemas de salud mental están dirigidas a contrarrestar los factores de riesgo y reforzar los factores protectores a lo largo de la vida, con el fin de modificar esos procesos que contribuyen a la enfermedad mental. Mientras más influencia tengan los factores individuales sobre el desarrollo de los trastornos mentales y la salud mental, mayor será el efecto preventivo que se pueda esperar cuando se implementen exitosamente. Es por ello que las intervenciones en edades precoces, que apuntan a la intervención en las ventanas de oportunidades del desarrollo, son tan relevantes y justifican con creces los costos.

Durante etapa prenatal y el desarrollo infantil, la reducción de la exposición a factores de riesgo ambientales, tales como toxinas y la deficiencia de micronutrientes, como el yodo, y la optimización de los servicios de salud pública para prevenir y tratar las enfermedades transmisibles, han permitido mejorar la atención obstétrica y prevenir complicaciones en el parto y lesiones del recién nacido.

Desde la infancia hasta la edad adulta, muchos factores de riesgo sociales y ambientales se concentran en las comunidades más pobres. El desarrollo de políticas de salud globales ayudaría a mejorar muchos de los determinantes sociales de la deficitaria salud mental que encontramos en diferentes regiones del mundo. Estos incluyen las políticas mundiales para abordar la escasez mundial de alimentos, de agua, tierra y energía, así como los esfuerzos mundiales para erradicar los regímenes opresivos y los conflictos armados.

En el ámbito nacional, la legislación para limitar y regular el acceso al alcohol y otras drogas; políticas económicas que promuevan una mayor equidad socioeconómica; políticas laborales que promuevan la seguridad del empleo y la mejora de las condiciones de trabajo, y políticas de bienestar que proporcionen la protección social a los enfermos, discapacitados y desempleados son algunos ejemplos de acciones que, de aplicarse, deberían tener un impacto positivo en la salud mental durante todo el ciclo vital(54).

Respecto de las intervenciones individuales y proximales, los estudios de evaluación económica sugieren que las intervenciones tempranas con niños y adolescentes ofrecen el mayor ahorro en los costos para las sociedades, mediante la reducción de los gastos futuros en salud(55).

Por otra parte, mientras las intervenciones universales son ideales para apoyar el desarrollo saludable para todos, en contextos de escasos recursos, las intervenciones selectivas e indicadas muestran efectos mayores y pueden ser más eficientes(56).

Durante el desarrollo prenatal y la infancia, los programas de visitas domiciliarias, como el disponible en el subsistema Chile Crece Contigo, son efectivos para los grupos vulnerables. Estos programas, que en su mayoría han sido realizados por enfermeras profesionales, pueden ser entregados como parte de los programas de atención primaria de la salud para mejorar, entre otras cosas, la salud mental de la madre, las habilidades parentelas y el apego. Dada la escasez de recursos profesionales en países de ingresos medio y bajo, se ha explorado la formación de individuos de la comunidad para ofrecer intervenciones similares, y la evidencia ha demostrado que es un enfoque eficaz, especialmente mediante el trabajo basado en pares y/o miembros de la comunidad que han padecido de una enfermedad mental(57). Durante la infancia y la adolescencia, la capacitación para los padres y los programas de aprendizaje socioemocional, especialmente los programas de habilidades para la vida -implementado en Chile desde hace casi 20 años-, han demostrado producir buenos retornos para la inversión en el largo plazo $(55,58)$.

El programa de intervención preventiva de Beardslee, desarrollado para familias en las que un padre o madre presenta depresión, y que busca prevenir la aparición de síntomas depresivos y otras patologías comórbidas asociadas en los hijos, ha demostrado tener buenos resultados a corto y largo plazo, y ha sido adaptado e implementado en diversos países, como EEUU, Colombia, Costa Rica, entre otros(59). Actualmente se encuentra en proceso de adaptación e implementación piloto en Chile (Proyecto Fondecyt No 11130615).

Los programas implementados en la educación preescolar y aquellos basados en la escuela han demostrado mejorar, entre otras cosas, el rendimiento académico del niño y sus relaciones socia- 
les, así como la participación de los padres en la educación, demostrando un efecto positivo en la promoción de la salud mental y la prevención de los trastornos conductuales y por uso de sustancias. La terapia cognitivo-conductual ha demostrado ser una estrategia eficaz para la prevención de la depresión en adolescentes con alto riesgo. Las intervenciones específicas para la prevención de la psicosis durante la adolescencia y la adultez temprana se han convertido en una estrategia prometedora, aunque su eficacia a largo plazo no está claramente establecida. En los adultos, la terapia cognitivo-conductual y la terapia interpersonal pueden promover el funcionamiento adaptativo y prevenir o retrasar la aparición de la depresión en grupos de riesgo para depresión. Los ejercicio de yoga y de alta energía puede mejorar el estado de ánimo y también ayudar a reducir los síntomas de ansiedad y depresión. Se han realizado iniciativas prometedoras en contextos de bajos recursos para hacer frente a los factores de estrés asociados a la pobreza y la exposición diferencial a los eventos vitales estresantes, como, por ejemplo, programas de fortalecimiento del capital social y de empoderamiento económico que promuevan una mayor equidad de género y sustentabilidad en grupos de riesgo. En los adultos mayores, el desarrollo de estrategias que incluyen la construcción de redes de apoyo para evitar el aislamiento social, así como la suplementación de ácido fólico y vitamina $B, y$ los ejercicios cognitivos han permitido prevenir la depresión y la demencia.

Algunos sectores, como la atención primaria de salud, la educación preescolar, la escuela y el lugar de trabajo, ofrecen oportunidades para la detección y las intervenciones tempranas en salud mental. Las intervenciones en estos ambientes exigen una respuesta multisectorial que involucren diversas disciplinas, como la medicina, la educación, la psicología, el trabajo social, la salud pública y la participación en la prestación de servicios. Dada la escasez de profesionales de todas las disciplinas en países de ingresos medio y bajo, la participación de personas de la comunidad local es esencial. Hay evidencia emergente de la eficacia de la delegación de funciones en las intervenciones de promoción de la salud mental por miembros capacitados de la comunidad(60).
El involucramiento de los miembros de la comunidad en el desarrollo y la entrega de servicios de salud mental tiene también el potencial de empoderarlos, mediante una mayor conciencia y capacidad para actuar sobre los determinantes sociales de la salud mental, generando así una mayor control de la comunidad sobre su salud. La delegación de funciones requiere, sin embargo, el desarrollo de materiales de apoyo técnico, así como la diversificación de los roles profesionales para asegurar un apoyo continuo y supervisión. Esto plantea un desafío tanto en países de ingresos altos como medios y bajos, dado que pocos programas de formación en las disciplinas relevantes en salud mental incluyen contenidos de promoción de la salud mental y prevención de las enfermedades mentales(56).

La extensión de la promoción de la salud y la prevención de las enfermedades requiere de actividades de abogacía y educación de los responsables políticos, la sociedad civil y los proveedores de servicios, sobre la necesidad y conveniencia de los esfuerzos en promoción y prevención en salud y los beneficios sociales que pueden traer los mismos.

Para este fin, la Organización Mundial de la Salud (OMS) desarrolló los Objetivos de Desarrollo del Milenio (ODM), que captaron la atención de la comunidad de salud internacional y el desarrollo cuando fueron lanzados hace 10 años(61). Los ODM proporcionan una visión de desarrollo en el que la salud y la educación están en el centro, pero curiosamente los objetivos de salud ignoran casi por completo las enfermedades no transmisibles, incluidos los trastornos mentales(62). A pesar de ello, existen tres ODM que están directamente relacionados con la salud mental: erradicación de la pobreza, reducción de la mortalidad infantil y mejora de la salud materna(48).

La meta de los ODM de salud estimuló el volumen global de la ayuda de la salud y dio lugar a un considerable aumento en la inversión y las acciones para enfermedades como la malaria y el VIH/ SIDA. La reducción de los problemas del VIH/ SIDA y la malaria fue exitosa porque el problema estaba claramente definido, los tratamientos fueron estandarizados y la estrategia estuvo basada en la investigación así como en la creciente con- 
ciencia del impacto y la carga producida por las enfermedades.

En los ODM, la salud mental estuvo ausente, pero el renovado interés global en la salud mental, que abarca la prevención y promoción de la salud, también permitió su consideración en los predecesores de los Objetivos de Desarrollo del Milenio (ODM), denominados los Objetivos de Desarrollo Sostenible (ODS). A fines de 2015 se logró definir como meta de los ODS la reducción en un tercio de la mortalidad prematura por enfermedades no transmisibles, mediante la prevención y el tratamiento, y promover la salud mental y el bienestar.

Los ODM han demostrado ser una poderosa herramienta para fortalecer los sistemas de salud en países de bajo y mediano ingreso, y por eso debemos aprender la lección e incluir la salud mental en los ODS posteriores a 2015. Los avances en VIH/SIDA y la malaria nos adelantan lo que se puede lograr en salud mental si es incluida en los ODS Post-2015. La agenda de la salud mental está directamente alineada con la agenda de desarrollo de la ONU y muchos de los objetivos de la organización tienen fuertes interdependencias para la salud mental. En efecto, el objetivo número 3 del Plan de acciones de Salud Mental 2013-2020 consiste en aplicar estrategias de promoción y prevención en materia de salud mental, implementando intervenciones psicosociales y comunitarias tempranas, científicamente contrastadas, sobre niños y adolescentes con trastornos mentales, con especial énfasis en la prevención del suicidio(24). Un mayor énfasis en la salud mental y la integración de la atención a la salud mental en todos los programas de desarrollo pertinentes fortalecerán el esfuerzo general de desarrollo, así como garantizará, por primera vez, la atención sostenida de la inversión y la investigación al principal contribuyente mundial de la pérdida de productividad y carga de enfermedad.

\section{Conclusiones}

El siglo XXI nos plantea un nuevo paradigma epistemológico en torno a las enfermedades mentales, entendiéndolas como enfermedades crónicas $(7,8)$, de alta prevalencia(2-5), con alto impacto en los AVISA(6), generando importan- te discapacidad(14-16) y alta morbimortalidad (por ejemplo, suicidio)(9-12). En este contexto, la alta morbimortalidad por enfermedades mentales no ha podido ser soslayada, pese a la disponibilidad de tratamientos efectivos y basados en la evidencia. Algunas de las brechas más estudiadas han sido en relación con la falta de acceso a tratamientos médicos (por ejemplo, psicofarmacológicos) y psicosociales (por ejemplo, psicoeducación, terapia cognitivo-conductual)(19-21) o la baja efectividad "en el mundo real" de los tratamientos psicofarmacológicos (por ejemplo, CATIE, STAR*D), debido a causas como la falta de adherencia a tratamiento farmacológico(28) y/o a la falta de efectividad inherente de estos tratamientos en algunas áreas psicopatológicas específicas (por ejemplo, antipsicóticos atípicos no han demostrado mejoría de síntomas cognitivos en esquizofrenia $(27,32)$, antidepresivos han demostrado solo un $20-30 \%$ de mejoría en la primera línea de tratamiento)(30,31). Por este motivo, y con el objetivo de reducir la morbimortalidad y discapacidad generada por las enfermedades mentales, se hace necesario un nuevo enfoque de prevención de enfermedades mentales y promoción de la salud mental $(18,38,43,52,53,55,56)$, especialmente debido a que un gran porcentaje de las patologías mentales crónicas comienzan en la infancia $(4,7,33,34,37)$ y están asociadas a factores de riesgo de enfermar(46-50). En un enfoque de promoción y prevención, las principales estrategias propuestas son: 1) detección de estados mentales de riesgo (por ejemplo, personas con síntomas subumbrales, familiares de primer grado con enfermedad mental, episodios breves y autolimitados; déficits neuropsicológicos precoces y rasgos específicos de personalidad premórbida; biomarcadores séricos, imagenológicos, bioeléctricos y genéticos de endofenotipos de enfermedades mentales específicas) $(34,37,40)$; 2) programas de promoción de estilos de estilos de vida saludables en salud mental (por ejemplo, ejercicio físico, estrategias de resolución de problemas, pensamientos positivos, toma de decisiones y psicoeducación para prevenir ansiedad y depresión en poblaciones vulnerables) (38,4042); 3) prevención de situaciones de riesgo que aumentan la probabilidad de enfermedad mental (por ejemplo, pobreza, aislamiento social, abuso y maltrato infantil, enfermedad mental perinatal 
de la madre, alimentación precaria del infante, acceso temprano a alcohol y drogas, inequidad socioeconómica e inestabilidad laboral, entre otros) (45-51), con énfasis en intervenciones tempranas a lo largo de la infancia - y todo el ciclo vitaly focalizadas en poblaciones de riesgo (especialmente en países de medianos y bajos ingresos) $(52,53,55)$.

Estas estrategias de abordaje permiten la implementación de intervenciones preventivas y de promoción de salud en la comunidad (escuela, lugar de trabajo, atención primaria en salud), como visita domiciliaria integral, trabajo basado en pares y delegación de funciones, capacitación de padres en escuelas, intervenciones preventivas orientadas a los hijos de familias con padres con enfermedad mental, entre otras(38,40-42,54-56). El objetivo es el empoderamiento de los sujetos más vulnerables en el abordaje sobre los determinantes sociales de su salud mental.

En el nivel de salud mental global, la incorporación de estos lineamientos en políticas públicas internacionales $(24,57,58)$ debiese guiar los objetivos propuestos localmente para las nuevas generaciones, en aras de reducir la prevalencia, morbimortalidad y discapacidad, hoy y a futuro, generada por las enfermedades mentales. 
Prevención e intervenciones tempranas en salud mental - Matías Irarrázaval, Fernanda Prieto, Julio Armijo

\section{Referencias}

1. Mercer A. Infections, Chronic Disease, and the Epidemiological Transition [Internet]. NED - New edition. Boydell \& Brewer; 2014. Available from: http://www.jstor.org/stable/10.7722/j.ctt6wp924

2. Steel Z, Marnane C, Iranpour C, Chey T, Jackson JW, Patel V, et al. The global prevalence of common mental disorders: a systematic review and meta-analysis 1980-2013. Int J Epidemiol 2014 Apr; 43(2): 476-493.

3. Vicente B, Saldivia S, de la Barra F, Kohn R, Pihan R, Valdivia M, et al. Prevalence of child and adolescent mental disorders in Chile: a community epidemiological study. J Child Psychol Psychiatry 2012 Oct; 53(10): 1026-1035.

4. Vicente B, Kohn R, Rioseco P, Saldivia S, Baker C, Torres S. Population prevalence of psychiatric disorders in Chile: 6-month and 1-month rates. Br J Psychiatry 2004 Apr; 184: 299-305.

5. Rodríguez JJ, Kohn R, Aguilar-Gaxiola S. Epidemiología de los trastornos mentales en América Latina y el Caribe. Washington: Organización Panamericana de la Salud; 2009: 1-343.

6. Whiteford HA, Degenhardt L, Rehm J, Baxter AJ, Ferrari AJ, Erskine HE, et al. Global burden of disease attributable to mental and substance use disorders: findings from the Global Burden of Disease Study 2010. Lancet 2013 Nov; 382(9904): 1575-1586.

7. Kessler RC, Berglund P, Demler O, Jin R, Merikangas KR, Walters EE. Lifetime prevalence and age-of-onset distributions of DSM-IV disorders in the National Comorbidity Survey Replication. Arch Gen Psychiatry; 2005 Jun; 62(6): 593-602.

8. Harding CM, Brooks GW, Ashikaga T, Strauss JS, Breier A. The Vermont longitudinal study of persons with severe mental illness, II: Long-term outcome of subjects who retrospectively met DSM-III criteria for schizophrenia. $A m J$ Psychiatry 1987 Jun;144(6): 727-735.

9. Mathers CD, Boerma T, Ma Fat D. Global and regional causes of death. Br Med Bull 2009; 92: 7-32.

10. Smink FRE, Van Hoeken D, Hoek HW. Epidemiology, course, and outcome of eating disorders. Curr Opin Psychiatry 2013 Nov; 26(6): 543-548.

11. Bertolote JM, Fleischmann A. Suicide and psychiatric diagnosis: a worldwide perspective. World Psychiatry 2002 Oct; 1(3): 181-185. Available from: http://www.ncbi.nlm.nih.gov/pmc/articles/PMC1489848/

12. Baxter AJ, Scott KM, Ferrari AJ, Norman RE, Vos T, Whiteford HA. Challenging the myth of an "epidemic" of common mental disorders: trends in the global prevalence of anxiety and depression between 1990 and 2010. Depress Anxiety 2014 Jun; 31(6): 506-516.

13. Kessler RC, Berglund P, Demler O, Jin R, Koretz D, Merikangas KR, et al. The epidemiology of major depressive disorder: results from the National Comorbidity Survey Replication (NCS-R). JAMA 2003 Jun; 289(23): 3095-3105.

14. WHO. World report on disability. Lancet 2011; 377(9782): 1977.

15. Jorquera N, Alvarado R, Libuy N, de Angel V. Association between Unmet Needs and Clinical Status in Patients with First Episode of Schizophrenia in Chile. Front psychiatry 2015; 6: 57.

16. Minoletti A, Zaccaria A. Plan Nacional de Salud Mental en Chile : 10 años de experiencia. Rev Panam Salud Pública 2005; 18(4/5): 346-358.

17. ONU. Convención sobre los Derechos de las Personas con Discapacidad. 2006; 1-35.

18. Insel TR, Scolnick EM. Cure therapeutics and strategic prevention: raising the bar for mental health research. Mol Psychiatry 2006 Jan; 11(1): 11-17.

19. McFarlane WR, Dixon L, Lukens E, Lucksted A. Family psychoeducation and schizophrenia: a review of the literature. J Marital Fam Ther 2003 Apr; 29(2): 223-245.

20. Resnick SG, Rosenheck RA, Dixon L, Lehman AF. Correlates of family contact with the mental health system: Allocation of a scarce resource. Ment Health Serv Res 2005; 7(2): 113-121.

21. Lehman AF, Steinwachs DM. Patterns of usual care for schizophrenia: initial results from the Schizophrenia Patient Outcomes Research Team (PORT) Client Survey. Schizophr Bull 1998; 24(1): 11-32.

22. WHO-AIMS. Report on mental health systems in Latin America and the Caribbean; 2013.

23. Access Economics Pty Limited. The economic impact of youth mental illness and the cost effectiveness of early intervention; 2009 (December).

24. WHO. Plan de acción sobre salud mental 2013-2020; 2013;54.

25. Ralph RO, Kidder KA. The recovery perspective and evidence-based practice for people with serious mental illness. Behav Heal Recover Manag Proj 2002 Jun.

26. Pilgrim D. Recovery From Mental Health Problems: Scratching The Surface Without Ethnography. J Soc Work Pract 2009 Dec; 23(4): 475-487. 
27. Young JW, Geyer MA. Developing treatments for cognitive deficits in schizophrenia: the challenge of translation. $J$ Psychopharmacol 2015 Feb; 29(2): 178-196.

28. Lieberman JA, Stroup TS, McEvoy JP, Swartz MS, Rosenheck RA, Perkins DO, et al. Effectiveness of antipsychotic drugs in patients with chronic schizophrenia. N Engl J Med 2005 Sep; 353(12): 1209-1223.

29. Emsley R, Chiliza B, Asmal L, Lehloenya K. The concepts of remission and recovery in schizophrenia. Curr Opin Psychiatry 2011 Mar; 24(2): 114-121.

30. Paykel ES. Partial remission, residual symptoms, and relapse in depression. Dialogues Clin Neurosci 2008; 10(4): 431437.

31. Trivedi MH, Rush AJ, Wisniewski SR, Nierenberg AA, Warden D, Ritz L, et al. Evaluation of outcomes with citalopram for depression using measurement-based care in STAR*D: implications for clinical practice. The American Journal of Psychiatry 2006: 28-40.

32. Depoortere R. Editorial: Current Approaches for Antipsychotics in Drug Discovery: Hope Beyond the Dopamine D2 Receptor? Current Pharmaceutical Design 2015: 3698-3699.

33. Birmaher B, Axelson D, Goldstein B, Strober M, Gill MK, Hunt J, et al. Four-year longitudinal course of children and adolescents with bipolar spectrum disorders: the Course and Outcome of Bipolar Youth (COBY) study. Am J Psychiatry 2009 Jul; 166(7): 795-804.

34. Reinherz HZ, Paradis AD, Giaconia RM, Stashwick CK, Fitzmaurice G. Childhood and Adolescent Predictors of Major Depression in the Transition to Adulthood. Am J Psychiatry 2003; 160(12): 2141-2147. Available from: http:// dx.doi.org/10.1176/appi.ajp.160.12.2141

35. Lindsay J, Laurin D, Verreault R, Hebert R, Helliwell B, Hill GB, et al. Risk factors for Alzheimer's disease: a prospective analysis from the Canadian Study of Health and Aging. Am J Epidemiol 2002 Sep; 156(5): 445-453.

36. Chamnan P, Simmons RK, Khaw KT, Wareham NJ, Griffin SJ. Estimating the potential population impact of stepwise screening strategies for identifying and treating individuals at high risk of Type 2 diabetes: a modelling study. Diabet Med 2012 Jul; 29(7): 893-904.

37. Corcoran C, Malaspina D, Hercher L. Prodromal interventions for schizophrenia vulnerability: the risks of being "at risk". Schizophr Res 2005 Mar; 73(2-3): 173-184.

38. Goldstein BL, Klein DN. A review of selected candidate endophenotypes for depression. Clin Psychol Rev 2014 Jul; 34(5): 417-427.

39. Clarke GN, Hawkins W, Murphy M, Sheeber LB, Lewinsohn PM, Seeley JR. Targeted Prevention of Unipolar Depressive Disorder in an At-Risk Sample of High School Adolescents: A Randomized Trial of a Group Cognitive Intervention. J Am Acad Child Adolesc Psychiatry 1995 Mar; 34(3): 312-21. Available from: http://www.sciencedirect.com/ science/article/pii/S0890856709637904

40. McGlashan TH, Zipursky RB, Perkins D, Addington J, Miller T, Woods SW, et al. Randomized, double-blind trial of olanzapine versus placebo in patients prodromally symptomatic for psychosis. Am J Psychiatry 2006 May; 163(5):790799.

41. Morrison AP, French P, Walford L, Lewis SW, Kilcommons A, Green J, et al. Cognitive therapy for the prevention of psychosis in people at ultra-high risk: randomised controlled trial. Br J Psychiatry 2004 Oct; 185: 291-297.

42. Asociación Americana de Psiquiatría. Guía de consulta de los criterios diagnósticos del DSM 5. 2013.

43. Bonhauser M, Fernandez G, Puschel K, Yanez F, Montero J, Thompson B, et al. Improving physical fitness and emotional well-being in adolescents of low socioeconomic status in Chile: results of a school-based controlled trial. Health Promot Int 2005 Jun; 20(2): 113-122.

44. Yu DL, Seligman MEP. Preventing depressive symptoms in Chinese children. Prev Treat 2002; 5(1).

45. Sharif F, Armitage P. The effect of psychological and educational counselling in reducing anxiety in nursing students. $J$ Psychiatr Ment Health Nurs 2004 Aug; 11(4): 386-392.

46. Osinowo HO, Olley BO, Adejumo AO. Evaluation of the effect of cognitive therapy on perioperative anxiety and depression among Nigerian surgical patients. West Afr J Med 2003 Dec; 22(4): 338-342.

47. Coie JD, Watt NF, West SG, Hawkins JD, Asarnow JR, Markman HJ, et al. The science of prevention. A conceptual framework and some directions for a national research program. Am Psychol 1993 Oct; 48(10): 1013-1022.

48. Patel V, Kleinman A. Poverty and common mental disorders in developing countries. Bull World Health Organ 2003; 81(8): 609-615.

49. Cacioppo JT, Cacioppo S. Social Relationships and Health: The Toxic Effects of Perceived Social Isolation. Soc Pers Psychol Compass 2014; 8(2): 58-72.

50. Patel V, Rahman A, Jacob KS, Hughes M. Effect of maternal mental health on infant growth in low income countries: new evidence from South Asia. BMJ 2004 Apr; 328(7443): 820-823.

51. Rahman A, Iqbal Z, Bunn J, Lovel H, Harrington R. Impact of maternal depression on infant nutritional status and 
Prevención e intervenciones tempranas en salud mental - Matías Irarrázaval, Fernanda Prieto, Julio Armijo

illness: a cohort study. Arch Gen Psychiatry 2004 Sep; 61(9): 946-952.

52. Ferri CP, Mitsuhiro SS, Barros MCM, Chalem E, Guinsburg R, Patel V, et al. The impact of maternal experience of violence and common mental disorders on neonatal outcomes: a survey of adolescent mothers in Sao Paulo, Brazil. BMC Public Health 2007; 7: 209.

53. Pearson RM, Evans J, Kounali D, Lewis G, Heron J, Ramchandani P, et al. Maternal depression during pregnancy and the postnatal period: risks and possible mechanisms for offspring depression at 18 years. JAMA Psychiatry 2013; 70(12): 1312-1319.

54. Patel V, Lund C, Hatherill S, Plagerson S, Corrigall J, Funk M, et al. Mental disorders: equity and social determinants. Equity, social determinants and public health programmes 2010; 115-134.

55. Zechmeister I, Kilian R, McDaid D. Is it worth investing in mental health promotion and prevention of mental illness? A systematic review of the evidence from economic evaluations. BMC Public Health 2008; 8: 20.

56. National Research Council (US) and Institute of Medicine (US) Committee on the Prevention of Mental Disorders and Substance Abuse Among Children, Youth and YARA and PI. Committee on the Prevention of Mental Disorders and Substance Abuse Among Children, Youth and Young Adults: Research Advances and Promising Interventions. Mary Ellen O' Connell, Thomas Boat and Kenneth E. Warner, Editors Board on Children, Youth. O'Connell ME, Boat T, Warner KE, editors. Washington, DC: National Academies Press; 2009.

57. Stastney P. Introducing peer support work in Latin American mental health services. Cad Saúde Colet 2012; 20(4): 473-481.

58. Knapp M, Mcdaid D, Parsonage M. Mental health promotion and mental illness prevention: The economic case. London: Department of Health; 2011.

59. Beardslee WR, Wright EJ, Salt P, Drezner K, Gladstone TR, Versage EM, et al. Examination of children's responses to two preventive intervention strategies over time. J Am Acad Child Adolesc Psychiatry 1997 Feb; 36(2): 196-204.

60. Barry MM, Clarke AM, Jenkins R, Patel V. A systematic review of the effectiveness of mental health promotion interventions for young people in low and middle income countries. BMC Public Health 2013; 13: 835.

61. Haines A, Cassels A. Can the millennium development goals be attained? BMJ 2004 Aug 12; 329(7462): 394-397. Available from: http://www.bmj.com/content/329/7462/394.abstract

62. Miranda JJ, Patel V. Achieving the Millennium Development Goals: Does Mental Health Play a Role? PLoS Med 2005; 2(10): e291. Available from: http://www.ncbi.nlm.nih.gov/pmc/articles/PMC1201694/

Recibido: 2 de febrero de 2016

Aceptado: 16 de febrero de 2016 